\title{
Im Spannungsfeld zwischen globalem Wandel und regionaler Dynamik. Die Großregionen Brasiliens
}

Martin Coy

Die Entwicklung Brasiliens während der letzten zehn Jahre wird gemeinhin als Erfolgsgeschichte gelesen. Spätestens mit der im Jahr 2003 von der führenden Investmentbank Goldmann Sachs veröffentlichten Studie "Dreaming with BRICs" gilt Brasilien endgültig als 'Land der Zukunft', als das es bereits Stefan Zweig in den 1940er Jahren bezeichnet hatte; nun allerdings aus der Sicht von und für Investoren, auf der Basis von Wachstumsziffern, wirtschaftlichen Potenzialen und Renditeerwartungen. Dabei sind es nicht nur erstaunliche Erfolge im industriellen und Dienstleistungssektor, die Brasilien in einem neuen Licht erscheinen lassen: Das Land ist inzwischen der drittgrößte Flugzeughersteller der Welt, nimmt eine wichtige Stellung im strategischen Bereich der KFZ-Produktion ein und überzeugt durch Spitzenforschung in den unterschiedlichsten Gebieten. Brasilien ist vor allem reich an Flächen und Rohstoffen. Flächenreichtum ist ein wichtiger Hintergrund für die führende Stellung des Landes auf den Weltagrarmärkten, und sowohl im Bereich vieler mineralischer als auch energetischer Rohstoffe kann Brasilien inzwischen seinen eigenen Bedarf decken und darüber hinaus als wichtiger Anbieter auf dem Weltmarkt auftreten. All dies zeigt, dass Brasilien - so wie allen großen Schwellenländern - in den kommenden Jahren eine Schlüsselstellung hinsichtlich seiner Positionierung im Globalen Wandel und damit bei der Frage des Umgangs mit der Anpassung an sowie der Bewältigung des Globalen Wandels zukommen wird. Anpassungs- und Bewältigungskapazitäten, was die Herausforderungen des Globalen Wandels anbelangt, hängen von den politischen Rahmenbedingungen, von den gesellschaftlich-institutionellen settings (Machtverhältnisse, Bereitschaft zum Wandel), vor allem aber auch von den sozioökonomischen, sozialkulturellen und ökologischen Potenzialen und Beschränkungen $\mathrm{ab}$, die sich räumlich verorten und in unterschiedlichen regionalen Dynamiken manifestieren. Dabei gilt für Brasilien, dass es von jeher durch wirtschaftliche, soziale und räumliche Disparitäten gekennzeichnet ist, in denen sich historisches Erbe, Entwicklungsstile und 
ihre Folgen, in jüngeren Jahren aber sicherlich auch die Konsequenzen der Einbindung des Landes in die Globalisierung niederschlagen. Insofern spielt das Spannungsfeld zwischen Globalem Wandel und regionaler Dynamik in allen Großregionen des Landes eine zunehmende Rolle. Vor diesem Hintergrund heißt "Brasilien verstehen", seine großregionale Struktur, Differenzierung und Dynamik in ihrer jeweiligen historischen, ökonomischen, sozialen und ökologischen Bedingtheit zu begreifen.

\section{Der Südosten: Zwischen Megaverstädterung und Wirtschaftslokomotive}

Das Herz Brasiliens schlägt im Südosten. So einfach könnte man es sich machen, wenn man die fünf brasilianischen Großregionen hinsichtlich der Indikatoren Bevölkerungsverteilung oder wirtschaftliche Wertschöpfung betrachtet. Nach dem letzten Bevölkerungszensus von 2010 leben $42 \%$ aller Brasilianer (das sind in absoluten Zahlen ca. 82 Millionen, also mehr als die Einwohnerzahl Deutschlands) in den vier Bundesstaaten des Südostens - São Paulo, Rio de Janeiro, Minas Gerais und Espírito Santo, die zusammen lediglich $11 \%$ der Gesamtfläche des Landes ausmachen. Die ebenfalls im Südosten liegenden drei größten städtischen Agglomerationen Brasiliens, São Paulo, Rio de Janeiro und Belo Horizonte und ihre jeweiligen Metropolitanregionen, stellen allein schon circa $20 \%$ der gesamten Bevölkerung des Landes. In den vier Bundesstaaten des Südostens werden $55 \%$ des Bruttoinlandsproduktes (2010) erwirtschaftet. Die herausragende Bedeutung der Region ist offensichtlich.

Naturräumlich wird der Südosten charakterisiert durch das Küstengebirge der Serra do Mar, die bis auf knapp $2.800 \mathrm{~m}$ ü.NN ansteigen kann. Ihr vorgelagert ist ein schmaler Küstenstreifen, der teilweise durch weiträumigere Küstenebenen erweitert wird. Im Hinterland geht das durch weite Täler, die wichtige Funktion für die Verkehrsverbindungen haben, gegliederte Küstengebirge in weit ausgedehnte Hochländer über, die als landwirtschaftliche Gunsträume von besonderer Bedeutung sind. Der Südosten Brasiliens war vor allem das Verbreitungsgebiet des Küstenregenwaldes, der Mata Atlântica, die, beginnend in der Kolonialzeit und seit dem 19. Jahrhundert rasant beschleunigt, der wirtschaftlichen ErschlieBung und 'Inwertsetzung' weichen musste. Man schätzt, dass heutzutage nur noch ca. 7 \% der ursprünglichen Mata Atlântica erhalten sind. 


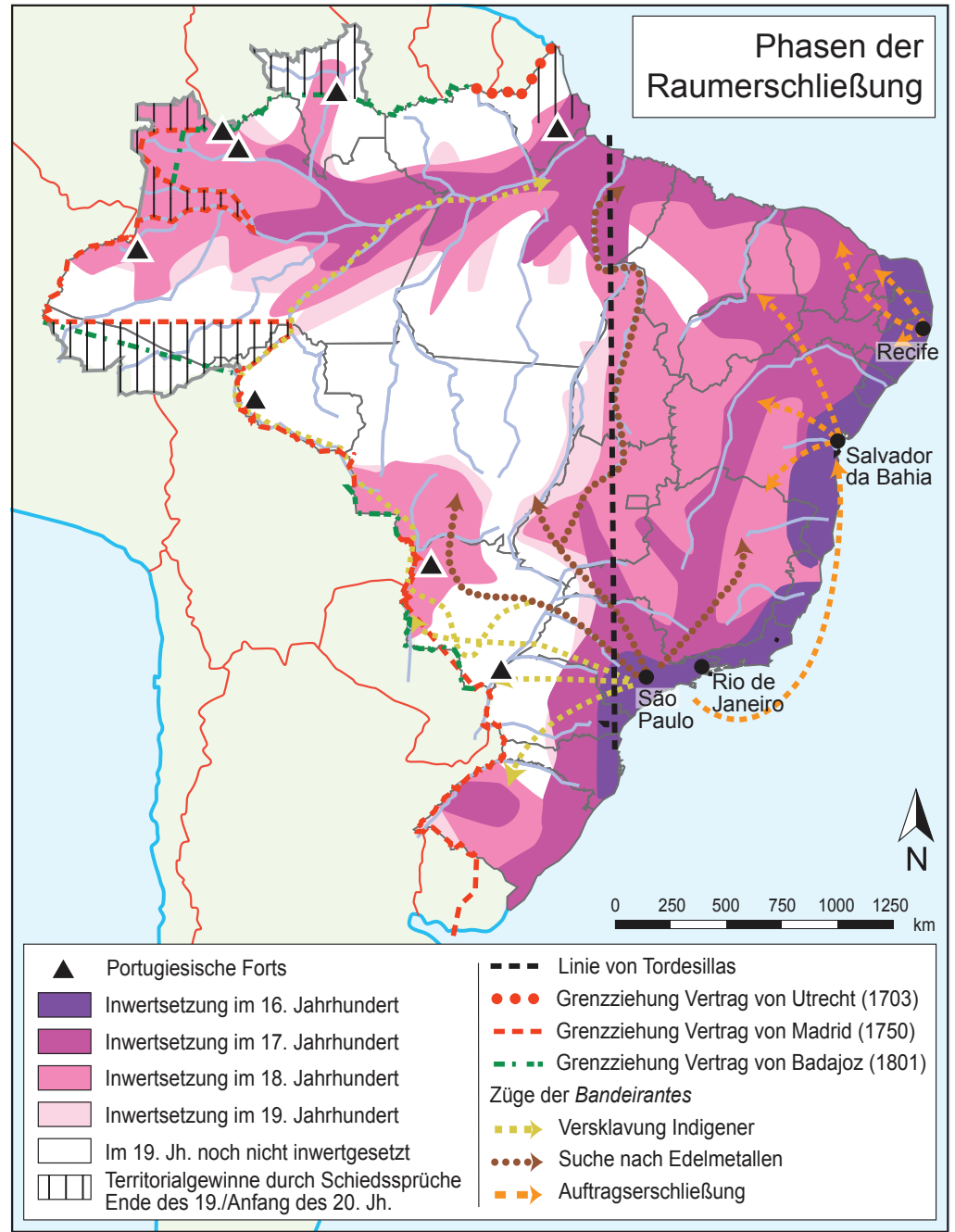

Entwurf: Hervé Théry, Überarbeitung: Tobias Töpfer

Quelle: Coy/Théry 2010

Nach dem Bergbauboom, der insbesondere Minas Gerais im 17. und 18. Jahrhundert zum begehrtesten Raum der portugiesischen Kolonie werden ließ, wurde ab dem 19. Jahrhundert der rasch expandierende Kaffeeanbau zum Motor des Aufstiegs des Südostens zur Kernregion Brasiliens. 
Zunächst noch auf der Basis von Sklavenarbeit organisiert, erhielten ab den letzten Jahrzehnten des 19. Jahrhunderts vor allem italienische Einwanderer entscheidende Bedeutung als Arbeitskräfte und Pächter in der Kaffeewirtschaft. Ausgehend von São Paulo dehnten sich zu Beginn des 20. Jahrhunderts die Kaffeepflanzungen entlang der sukzessive ausgebauten Eisenbahnlinien immer weiter in das Hinterland aus.

Das mit dem Kaffee erwirtschaftete Kapital wurde in den bereits existierenden und entlang der Eisenbahnen neu entstehenden Städten zunehmend in gewerbliche und industrielle Aktivitäten investiert. Insofern kann man mit Fug und Recht sagen, dass die Industrieregion SüdostBrasilien ihren wesentlichen Ursprung im Kaffeeanbau hat. Dies gilt vor allem auch für den Standort São Paulo. Zunächst auf der Verarbeitung landwirtschaftlicher Produkte und der Textilindustrie fußend, trat der Südosten in den 1940er Jahren mit dem seitens der USA mitfinanzierten Aufbau des ersten großen Schwerindustriekomplexes in Volta Redonda im Bundesstaat Rio de Janeiro massiv in das Industriezeitalter ein. In den 1950er Jahren, in der Modernisierungsphase der brasilianischen Wirtschaft und Gesellschaft, die mit dem Präsidenten Juscelino Kubitschek wie mit keinem anderen verbunden ist, erweiterte und diversifizierte sich das industrielle Branchenspektrum des Südostens erheblich. Vor allem Minas Gerais wurde auf der Basis der dortigen Eisenerz- und Manganvorkommen zum wichtigsten Eisen- und Stahlstandort Brasiliens. Die Zeche zahlte allerdings die Umwelt, denn in Ermangelung von Steinkohle wurde die Mata Atlântica als Lieferant von Holzkohle für die Schwerindustrie missbraucht. Vor allem São Paulo und sein Umland, aber auch die Großräume Belo Horizonte und Rio de Janeiro, wuchsen in der zweiten Hälfte des 20. Jahrhunderts zu Industrieagglomerationen von nationaler und internationaler Bedeutung heran. Der Fahrzeugbau, die Elektrotechnik und Elektronikindustrie, Chemie und Petrochemie (hier vor allem der wegen seiner Umweltbelastungen berühmt-berüchtigte Standort $\mathrm{Cu}$ batão in der Baixada Santista) sowie in jüngerer Zeit Flugzeugbau, Raumfahrtindustrie sowie andere Schlüsseltechnologien kennzeichnen die industrielle Vielfalt des brasilianischen Südostens. São José dos Campos, zwischen São Paulo und Rio de Janeiro im Vale do Paraíba gelegen, ist unter anderem als Standort von EMBRAER, dem inzwischen drittgrößten Flugzeughersteller weltweit, vielleicht am ehesten als 'symbolischer Ort' der Transformation dieser Großregion zum Hochtechnologiestandort anzusehen. 
Trotz der nach wie vor bestehenden Vormachtstellung des Südostens lässt sich in den letzten Jahren ein deutlicher industrieller Dekonzentrations- und Standortverlagerungsprozess beobachten. Beherbergte die Großregion 1996 noch mehr als 60 \% aller Industriebetriebe des Landes, waren es 2010 (wenn auch auf absolut sehr viel höherem Niveau) 'nur' noch etwa $47 \%$. 'Gewinner' sind aufgrund steuerlicher Vergünstigungen und sonstiger komparativer Kostenvorteile insbesondere der Süden und Nordosten.

Trotz aller Vorherrschaft der modernen Industrie für die großregionale Beschäftigung und Wertschöpfung bleibt der Südosten nach wie vor auch ein wesentlicher Standort des Agrobusiness. Auch wenn der Kaffeeanbau bis auf den heutigen Tag eine wichtige Rolle spielt, wurde er in vielen Regionen doch von seiner ehemaligen Monopolstellung verdrängt. In erster Linie hat sich der großbetriebliche Zuckerrohranbau in weiten Teilen von São Paulo im Gefolge der staatlich geförderten Herstellung von Ethanol als Biotreibstoff seit den 1970er Jahren durchgesetzt. Die großen Usinas bestimmen nicht nur den ländlichen Raum, sondern indirekt auch zahlreiche Landstädte, an deren Peripherien das 'Arbeitsheer' der bóia-fria, der Tagelöhner der Zuckerrohrbetriebe, ein Überleben zu sichern versucht. Ein weiterer, stark exportorientierter Faktor des südostbrasilianischen Agrarsektors ist der Anbau von Zitrusfrüchten. Auch in diesem Sektor sind erhebliche agrarsoziale Disparitäten zu beobachten. Schließlich ist der Südosten Brasiliens - und dies gilt für große Teile sowohl von São Paulo als auch von Minas Gerais - eine Region der Rinderweidewirtschaft, die auf großen Fazendas betrieben wird. Vor diesem Hintergrund ist es nicht verwunderlich, dass es hier auch immer wieder zu Landkonflikten gekommen ist. In den letzten Jahren ist die Großregion zu einem wichtigen Aktionsgebiet der Landlosenbewegung MST (Movimento dos Trabalhadores Sem-Terra) geworden (als 'emblematische' Region ist in diesem Zusammenhang Pontal do Paranapanema, das Grenzgebiet zu Paraná, zu nennen).

Der Strukturwandel in der Landwirtschaft mit seiner Verdrängungswirkung und vor allem die Dynamik des industriellen Sektors sind wesentliche driving forces eines rasanten Verstädterungsprozesses, der im Laufe des 20. Jahrhunderts zum Charakteristikum des räumlichen Wandels des brasilianischen Südostens wurde. Offiziell sind mehr als $93 \%$ der Bevölkerung des Südostens städtisch. Dabei ziehen die beiden Megastädte São Paulo und Rio de Janeiro von jeher die größte Aufmerksamkeit 
auf sich. Die Metropolregion von São Paulo gilt mit inzwischen mehr als 20 Millionen Einwohnern als sechstgrößte weltweit. Rio de Janeiro folgt mit einer Einwohnerzahl in der Metropolregion von ca. 12 Millionen auf dem 24. Platz im weltweiten Vergleich. Mit São Paulo und Rio verfügt

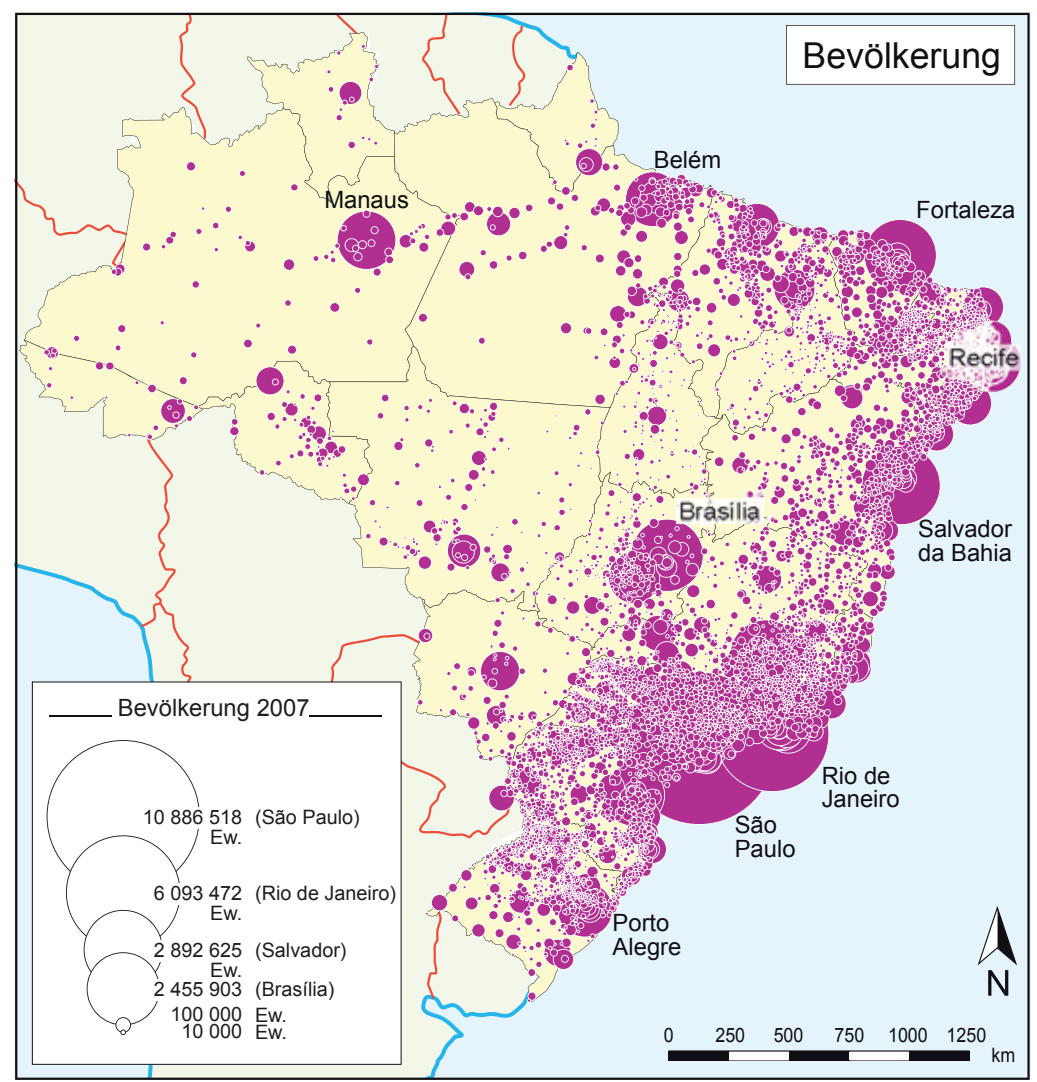

Entwurf: Hervé Théry, Überarbeitung: Tobias Töpfer

Quelle: Coy/Théry 2010

der brasilianische Südosten über zwei der insgesamt vier megaurbanen Agglomerationen (mehr als 10 Millionen Einwohner) Lateinamerikas. Bei aller Dominanz der beiden Megastädte (zusammen mit der über fünf Millionen Einwohner zählenden Agglomeration Belo Horizonte) übersieht 
man leicht, dass gerade im brasilianischen Südosten Verstädterung mehr meint als Mega-Verstädterung. Gerade Groß- und Mittelstädte sowohl im weiteren Einflussgebiet der Metropolräume als auch in den periphereren ländlich geprägten Räumen prägen die Vielfalt des Städtischen in dieser Großregion und gehören in vielen Fällen zu den besonders dynamischen urbanen Räumen. Musterbeispiel dafür ist vielleicht Campinas, die Millionenstadt im weiteren Umfeld von São Paulo, Standort hochrangiger Forschungseinrichtungen und Technologieunternehmen, die den Wandel Brasiliens und des Südostens besonders gut repräsentiert.

Die beiden Megastädte São Paulo und Rio de Janeiro sind in ihrer Struktur Vorreiter und Musterbeispiele für den Prozess der sozialräumlichen Fragmentierung, der die Stadtentwicklung und den städtischen Wandel in allen lateinamerikanischen Ländern während der letzten Jahrzehnte prägt. Oftmals in unmittelbarer Nachbarschaft finden sich Favelas mit den in Brasilien als condominios fechados bezeichneten Reichenghettos. Beispiele hierfür sind Barra da Tijuca, das Erweiterungsgebiet der noblen Zona Sul von Rio de Janeiro, oder der in direkter Nachbarschaft zur Favela Paraisópolis gelegene Privilegiertenstadtteil Morumbi in São Paulo. Allein in São Paulo existieren über 50 Shopping Center, die als 'Konsum- und Freizeitenklaven' zunehmend die Kommunikations- und Repräsentationsfunktionen des öffentlichen Raumes übernehmen. Die Innenstadtbereiche durchliefen in den letzten Jahrzehnten tief greifende Veränderungen. Die öffentlichen Räume wurden zunehmend zu Überlebensräumen marginalisierter Bevölkerungsgruppen (informeller Straßenhandel, Obdachlose etc.). Die Aufenthaltsqualität in den Innenstädten verringerte sich, unter anderem infolge ihrer sukzessiven Zurichtung auf den motorisierten Individualverkehr. Auch als Standort hochrangiger Dienstleistungen mussten die Innenstädte, insbesondere das Zentrum von São Paulo, erhebliche Einbußen hinnehmen. Finanzsektor, Firmenzentralen, Niederlassungen ausländischer Unternehmen und unternehmensorientierte Dienstleister wandern seit Jahren in einen südwestlichen Sektor der Kernstadt ab, wo sich hochmoderne Bürostandortkonzentrationen herausgebildet haben, die die wachsende Bedeutung São Paulos als global vernetzte 'Steuerungszentrale' in der gebauten Umwelt symbolisiert anzeigen. Im Gefolge dieses Verlagerungsprozesses von Steuerungsfunktionen erhalten Versuche einer Revitalisierung der Innenstadt eine zunehmende Bedeutung. Bahnhöfe und sonstige öffentliche Gebäude werden saniert und zu Museen, Konzertsälen oder Kulturzentren umgebaut, öffentliche Räume werden neu gestal- 
tet. Dabei kommt es nur allzu oft zu einer Verdrängung derjenigen, für die das Stadtzentrum inzwischen Überlebensfunktion erhalten hat. Diese sozialräumliche Ambivalenz von Stadterneuerung zeigt sich im Moment vor allem in der Vorbereitung auf die Groß-Events der nächsten Jahre: die Fußballweltmeisterschaft 2014 und die Olympischen Spiele 2016. Dabei sind Stadion-Um- und -Neubauten oder die Erstellung der olympischen Sportstätten nur ein Aspekt. Die brasilianischen Städte - und besonders Rio de Janeiro - nutzen die Großsportereignisse zum groß angelegten Stadtumbau, der, stark auf dem Gedanken von public-private-partnerships fuBend, im Sinne einer 'unternehmerischen Stadtpolitik' durchgeführt wird, für die Investoreninteressen, Standortqualität, Stadtimage und Stadtmarketing prioritär sind. Dagegen treten Vorstellungen von einem 'Recht auf Stadt' für alle, die sich in Brasilien im Zuge einer nationalen Stadtreformbewegung ab den 1980er Jahren durchaus etablieren konnten, zusehends in den Hintergrund. Wie unter dem Brennglas kann dies derzeit bei der Realisierung des Stadterneuerungsprojektes Porto Maravilha in Rio de Janeiro beobachtet werden. Werden also auf Dauer die bestehenden Ungleichheiten in Brasilien auf den unterschiedlichen Maßstabsebenen eher verringert oder weiter verstärkt? Die derzeitigen Entwicklungstrends im Zuge der bevorstehenden Megaevents weisen zumindest nicht auf einen Wirkung versprechenden Abbau von Disparitäten hin.

\section{Der Nordosten: Perspektiven für das 'Armenhaus' Brasiliens?}

Die neun Bundesstaaten der Nordostregion (Maranhão, Piauí, Rio Grande do Norte, Ceará, Paraíba, Pernambuco, Alagoas, Sergipe und Bahia) stellen mit ca. 54 Millionen Einwohnern die zweitgrößte Bevölkerungszahl des Landes. Beim ersten nationalen Zensus Brasiliens im Jahr 1872 war der Nordosten noch die bevölkerungsreichste Region, seitdem verliert er jedoch durch Abwanderung an Gewicht. Eine Reihe von sozialen Indikatoren kennzeichnen den Nordosten heute als Problemregion: Das Pro-Kopf-Einkommen ist mit ca. 9.600 Reais (2010) das niedrigste Brasiliens und erreicht gerade einmal $40 \%$ des Wertes für den Südosten. Der HDI-Wert (Human Development Index) des Nordostens ist mit 0,72 der niedrigste im Vergleich der Großregionen, die Analphabetenquote mit ca. $17 \%$ und die Säuglingssterblichkeit mit 19\% sind die höchsten im gesamtbrasilianischen Vergleich. 
Natur- und wirtschaftsräumlich lässt sich der Nordosten in drei mehr oder minder parallel zur Küste verlaufende Raumeinheiten untergliedern: Die Küstenebene der Zona da Mata, ausgestattet mit vergleichsweise fruchtbaren Böden und mit ausreichenden Niederschlagsmengen versorgt. Hier fand nach der Ankunft der Portugiesen ab dem 16. Jahrhundert die Ausbreitung der Zuckerrohrplantagen beste Voraussetzungen, und bis heute spielt der Zuckerrohranbau in vielen Bereichen der Zona da Mata (im Süden von Bahia auch der Kakaoanbau) eine große Rolle. Die Küstenregion ist als historischer Kernraum des Nordostens auch der Bevölkerungsschwerpunkt. Hier konzentrieren sich die Hauptstädte der Bundesstaaten und sonstigen Regionalmetropolen. An das Küstentiefland schließt sich der vergleichsweise schmale Streifen des Agreste an, ein 'Übergangsraum', in dem eine teilweise recht intensive landwirtschaftliche Nutzung (Versorgung der Metropolitanräume an der Küste) zu beobachten ist, die durch ausreichende Niederschläge ermöglicht wird. Ein Band von Mittelstädten übernimmt hier seit jeher eine 'Drehscheibenfunktion' für den Handel zwischen Küstenraum und Hinterland sowie eine industrielle Produktionsfunktion, beispielsweise im Bereich der Textilindustrie. Der Agreste geht schließlich in die Weiten des Sertão, des klimatisch vorwiegend semiariden Hinterlandes, über, das flächenmäßig den größten Teil der Nordostregion ausmacht. Das Ausbleiben der Niederschläge führt immer wieder zu Dürreperioden, die seit jeher die Abwanderung großer Bevölkerungsmengen zur Folge haben. Aber es ist keineswegs nur die naturräumliche 'Benachteiligung', die den Nordosten zur Problemregion des Landes machen. Die wirtschaftlichen und vor allem die sozialen Verhältnisse üben mindestens ebenso eine strukturell bedingte Verdrängungswirkung aus. Im Hinterland des Sertão herrscht, von den wenigen klein- und mittelstädtischen Zentren abgesehen, im Wesentlichen eine extensive Viehhaltung vor. Ausgedehnte Fazendas, die sich oftmals nach wie vor in den Händen der traditionellen Agraroligarchien befinden, stehen kleinbäuerlicher Subsistenzlandwirtschaft entgegen. Diese seit jeher bestehenden (agrar)sozialen Disparitäten sind mit ungleichen Zugangs- und Verfügungsrechten bezüglich der überlebenswichtigen Ressourcen (Land, Wasser etc.), generell mit ungleichen Machtverhältnissen und ungleichen Überlebenschancen verbunden. Temporäre oder dauerhafte Abwanderung wird somit zur weithin bestimmenden Überlebensstrategie.

Insgesamt gilt der Nordosten als die Region Brasiliens, in der das 'koloniale Erbe' am stärksten präsent ist. Die ehemalige Dominanz der 
Sklaverei als Basis der kolonialen Plantagenwirtschaft drückt sich nach wie vor in der ethnischen Zusammensetzung der Bevölkerung aus (im nationalen Vergleich die höchsten Anteile schwarzer Bevölkerung), traditionelle Machtstrukturen und coronelismo pausen sich bis heute in politischem Klientelismus und der Dominanz der alten regionalen Eliten durch. Armut als Folge von wirtschaftlichem Niedergang bzw. wirtschaftlicher Stagnation und persistenter Ungleichheit wird zur wesentlichen Triebkraft der Verdrängungsmigration, die dazu führt, dass nordestinos sowohl das Arbeitskräftereservoir in den Megastädten des Südostens bilden als auch an den Siedlungsgrenzen Amazoniens seit jeher als Kautschukzapfer, Kleinbauern oder aber als garimpeiros (illegale Goldsucher und Diamantenschürfer) eine wesentliche Rolle spielen.

Aber das Bild von der Rückständigkeit und Krisenhaftigkeit des Nordostens ist bei genauerer Betrachtung nur die halbe Wahrheit. Mindestens seit einem halben Jahrhundert steht besonders der Nordosten im Zentrum der Bemühungen um Regionalentwicklung und 'Modernisierung'. Die Gründung der SUDENE (Superintendência do Desenvolvimento do Nordeste) in den späten 1950er Jahren gilt als der emblematische Startpunkt einer zentral gesteuerten Regionalentwicklungspolitik, die weit über Brasilien hinaus Beachtung fand. Schon früher hatte der Zentralstaat seine Anstrengungen zur Dürrebekämpfung in Kampagnen und Behörden institutionalisiert, deren Wirkungsgrad allerdings umstritten war. Im Zuge der allgemeinen Modernisierungsbemühungen ab den 1970er Jahren verstärkte der Staat seine Politik der Regionalentwicklung im Nordosten. Im Zentrum stand der Bau von Staudämmen und Wasserkraftwerken entlang des Rio São Francisco. Dort entstanden auch im Grenzgebiet zwischen Bahia und Pernambuco, um die beiden Städte Petrolina und Juazeiro, mit der Fertigstellung des Sobradinho-Staudamms die großen Bewässerungsoasen, die heute als Produktionsgebiete von tropischen Früchten (vor allem Mango und Papaya) sowie von Tafeltrauben voll und ganz in globale Wertschöpfungsketten integriert sind. Seit geraumer Zeit ist im Nordosten auch ein zunehmendes Bemühen um Industrieansiedlung zu beobachten. In jüngeren Jahren befinden sich die Bundesstaaten des Nordostens (neben Bahia vor allem Ceará und Pernambuco) unter Mithilfe von Steuervergünstigungen und vergleichsweise niedrigen Produktionskosten (vor allem Lohnkosten) durchaus mit Erfolg in einem heftigen Standortwettbewerb mit den Bundesstaaten des Südens und Südostens hinsichtlich der Ansiedlung neuer Produktionsstätten nationaler und internationaler 
Unternehmen. Der Ausbau der regionalen Infrastruktur soll die Standortbedingungen im Nordosten verbessern und dessen Einbindung in globale Märkte verstärken. Hierbei spielen vor allem neue Häfen, insbesondere Suape im Großraum Recife und Pecém im Großraum Fortaleza, eine strategische Rolle. Eine Anbindung des Hinterlandes an diese neuen Großhäfen beispielsweise mittels neuer Eisenbahnen ist geplant oder befindet sich bereits in Umsetzung.

Von einem Großprojekt, dessen Idee bereits im 19. Jahrhundert geboren wurde und das vor allem die Regierung Lula mit großem Nachdruck betrieben hat, versprechen sich die Einen eine nachhaltige Lösung der Probleme des Sertão, die Anderen befürchten eher eine Verstärkung von sozialen Ungleichheiten. Die Rede ist von der Transposição do Rio São Francisco, der Ableitung von Flusswasser aus dem Rio São Francisco zur Versorgung weiter Teile des Hinterlandes von Pernambuco, Ceará, Rio Grande do Norte und anderen Bereichen. Mit diesem seit 2007 betriebenen Großprojekt, das unter anderem Priorität im Rahmen des nationalen Investitionsprogramms PAC (Programa de Aceleraşão para o Crescimento) der Regierungen Lula und Rousseff hat, soll Wasser aus dem Rio São Francisco in zwei Ableitungskanälen in die Trockengebiete geleitet werden. Die Befürchtungen gehen dahin, dass sich auch im Rahmen dieses Großprojektes die Tendenz hin zu einer "Kommodifizierung" des Allmendegutes Wasser verstärken wird, dass in diesem Zusammenhang die Kapitalkräftigen - beispielsweise über die Ausdehnung von Bewässerungsoasen - die Gewinner des Projektes sind, die eigentlich Bedürftigen aber eher leer ausgehen werden. $\mathrm{Ob}$ von diesem wie von vielen anderen Projekten wirklich neue Perspektiven für den Nordosten Brasiliens ausgehen, wird die Zukunft zeigen. Skepsis bleibt angebracht, ob sich im Endeffekt nicht doch die tief in die gesellschaftlichen und räumlichen Strukturen der Großregion eingeschriebenen Ungleichheiten reproduzieren oder sogar noch verstärken.

\section{Der Norden: Zwischen Regenwaldzerstörung und nachhaltiger Entwicklung}

Amazonien gehört zu jenen Regionen Brasiliens, die in besonderem Maße ganz unterschiedliche Assoziationen auslösen. Region der Sehnsüchte nach dem arkadischen Leben der 'Wildnis', geheimnisvolle Region des Unbekannten, gleichzeitig aber auch 'Grüne Hölle' des undurchdringli- 


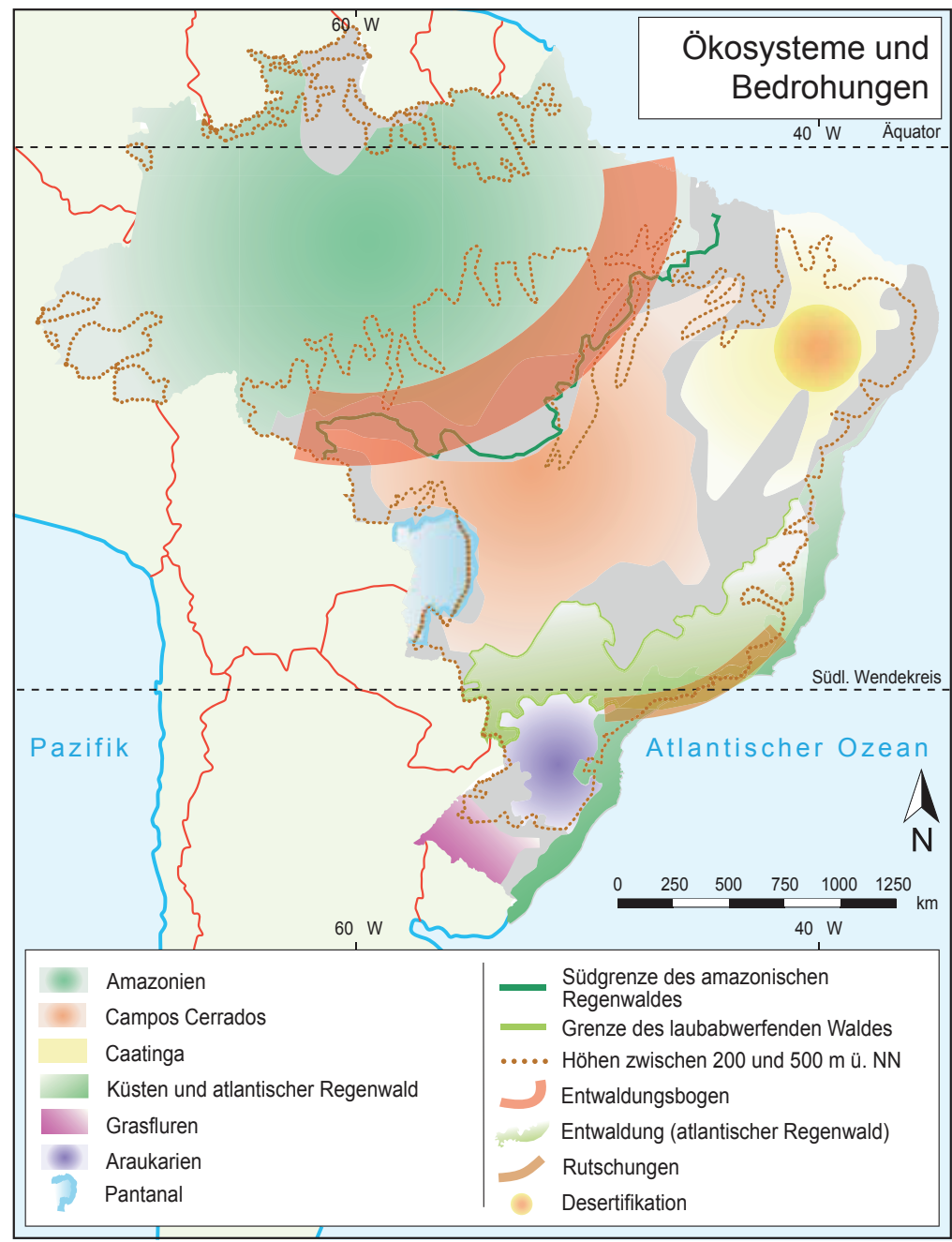

Entwurf: Hervé Théry, Überarbeitung: Tobias Töpfer

Quelle: Coy/Théry 2010

chen 'Dschungels' und der bedrohlichen Gefahren. In jedem Fall ist Amazonien, die riesige Peripherie im Norden Brasiliens, seit jeher ein Ort der Mythen, ein die Phantasie beflügelnder Raum und eigentlich eine bis auf den heutigen Tag in weiten Teilen unbekannte Region. 
Amazonien ist nach wie vor mit ca. 4 Millionen $\mathrm{km}^{2}$ das größte zusammenhängende tropische Regenwaldgebiet der Erde, auch wenn in den letzten 40 Jahren ca. $15 \%$ der Regenwaldfläche zerstört oder zumindest stark degradiert wurden, der größte Teil davon in Brasilien. Die genaue Länge des Amazonas anzugeben, ist schwierig. Je nach Quellfluss dürfte sie zwischen 6.500 und $6.800 \mathrm{~km}$ liegen. Somit kann sich der Amazonas mit dem Nil um den Titel des längsten Flusses unserer Erde streiten. Unumstritten ist, dass der Amazonas zusammen mit seinen mehr als 1.000 Zuflüssen das sich über eine Fläche von ca. 6,8 Millionen $\mathrm{km}^{2}$ erstreckende größte Flusseinzugsgebiet der Erde bildet und zwischen 10 und $15 \%$ des gesamten Süßwassers der Erde dem Atlantischen Ozean zuführt auch dies ein Weltrekord. Die globale Bedeutung des Naturraums Amazonien wird also schon aus diesen wenigen Superlativen offensichtlich, auch deshalb kein Wunder, wenn in jüngeren Jahren angesichts des Globalen Wandels der Blick der Weltöffentlichkeit immer wieder auf das Gebiet gerichtet ist.

Fast zwei Drittel Amazoniens gehören zu Brasilien, und das Gebiet stellt fast $60 \%$ des brasilianischen Staatsterritoriums dar. Aber was ist eigentlich gemeint, wenn von Amazonien die Rede ist? Das Flusseinzugsgebiet, der Natur- oder Landschaftsraum (in Brasilien ca. 4,1 Mio. $\mathrm{km}^{2}$ umfassend), das 'statistische' Amazonien (die Nordregion mit den brasilianischen Bundesstaaten Acre, Amapá, Amazonas, Pará, Rondônia, Roraima und Tocantins) oder die seit den 1950er Jahren bestehende brasilianische Planungsregion Amazônia Legal, die 5,5 Mio. $\mathrm{km}^{2}$ umfasst und zu der, zusätzlich zu den bereits genannten Bundesstaaten, noch Mato Grosso und Teile des Bundesstaates Maranhão gehören. In dieser Region Amazônia Legal leben nach dem Zensus von 2010 ca. 24 Millionen Menschen, das entspricht lediglich $12 \%$ der Gesamtbevölkerung Brasiliens auf $61 \%$ des Gesamtterritoriums des Landes. Allerdings konzentrieren sich dort mit 250.000 Bewohnern fast $60 \%$ der indigenen Bevölkerung Brasiliens. Insgesamt wenig Menschen in einem riesigen Raum, zumal inzwischen über $70 \%$ aller Bewohner Amazoniens in den Städten der Region leben.

Schon immer zog Amazonien die unterschiedlichsten Begehrlichkeiten der Menschen in besonderem Maß auf sich. Im 16. Jahrhundert suchten die Spanier dort das sagenumwobene Eldorado. Auch in den folgenden Jahrhunderten war der Entdeckungsdrang zumeist mit der Hoffnung auf Reichtum oder zumindest wirtschaftlich Verwertbares verbunden. Die extrem große Biodiversität Amazoniens - eine der höchsten 
weltweit - spielte dabei von jeher eine zentrale Rolle. So sind auch die verschiedensten Kulturpflanzen aus Amazonien über die ganze Welt verbreitet worden. Das bekannteste Beispiel dafür ist der Kautschukbaum, Hevea brasiliensis. Unmittelbar mit dem Industrialisierungsprozess im 19. Jahrhundert verbunden, hat diese Pflanze Amazonien gegen Ende des 19. und zu Beginn des 20. Jahrhunderts einen Boom beschert, der die extreme Peripherie kurzzeitig ins weltweite Rampenlicht stellte. Die großen Städte der Region, Belém und Manaus, wurden mit den Symbolen des Wohlstands, der Kultur und der Entwicklung ausgestattet: Opernhäuser, Theater, elektrische Straßenbeleuchtung, Straßenbahnen. In die Region wanderten Menschen aus den Armutsgebieten Brasiliens in der Hoffnung auf ein besseres Leben zu. Allerdings endete der kurzzeitige Boom nach wenigen Jahren abrupt, und die Region versank wieder weitgehend in die Stagnation der Peripherie.

Der Zweite Weltkrieg brachte zwar nochmal ein kurzes Wiederaufleben des Kautschukbooms, im Grunde genommen dauerte es aber bis in die 1960er und 1970er Jahre, um Amazonien erneut in den Fokus zu bringen. Grund war nun eine im Wesentlichen durch den Staat ausgelöste und bis heute anhaltende Regionalentwicklungsdynamik, die in weiten Teilen der Region tief greifende demographische, wirtschaftliche, soziale, kulturelle, vor allem aber auch ökologische Veränderungen in einem zuvor unbekannten Ausmaß mit sich brachte. Straßenbau, kleinbäuerliche Agrarkolonisation, großbetriebliche Rinderweidewirtschaft, Holzeinschlag, die Ausbeutung mineralischer Ressourcen, der Ausbau des hydroenergetischen Potenzials, dies sind nur die wichtigsten Faktoren dafür, dass Amazonien als soziales Sicherheitsventil, aber auch als schier grenzenloser 'Ergänzungsraum' für die brasilianische Wirtschaft und als neue Ressourcenfrontier galt. Verbunden sind damit zahlreiche Konflikte um unterschiedliche Nutzungsinteressen und Überlebensstrategien, um Land vor allem, aber auch um die politische Vorherrschaft und um die geostrategische Kontrolle, die allzu oft gewaltsam ausgetragen werden. Die neue Regionalentwicklungsdynamik der letzten Jahrzehnte brachte eine völlige Kehrtwende hinsichtlich der Schwerpunkträume von Bevölkerung und wirtschaftlicher Entwicklung innerhalb Amazoniens mit sich. Waren es zuvor vor allem die Bereiche der Várzea (s. u.) und insgesamt die Regionen an den Flüssen, die als die Gunsträume und entwickelte Gebiete galten, so kehrt die Regionalentwicklung der letzten Jahrzehnte diesen Räumen eher den Rücken zu. Die Flüsse als Lebensadern, die die Überlebensstra- 
tegien, die Ernährungsgewohnheiten, Kommunikation und Handel, kurz den Lebensstil der Menschen über Jahrhunderte bestimmten, werden von den neuen Fernstraßen abgelöst, die Amazonien aus seiner - vermeintlichen - Isolation herauslösen, die Peripherie an die Zentrumsregionen des Südostens und Südens anbinden, eine massive Zuwanderung überhaupt erst ermöglichen und die letztendlich eine völlig neue Siedlungsstruktur bewirken. Nun sind es die Pionierstädte an den Straßen und nicht mehr die traditionellen Flusshäfen, die die wirtschaftlichen, politischen und all$\mathrm{zu}$ oft auch die neuen kulturellen Zentren der Region bilden.

Die von den Militärs in der Zeit der Diktatur ab den 1960er Jahren unter dem Motto "Land ohne Menschen für Menschen ohne Land" betriebene Erschließung Amazoniens sollte die vormalige Peripherie in den beschleunigten Rhythmus der Modernisierung einbinden. Die Interessen der rechtmäßigen Bewohner Amazoniens, der Indigenen, der caboclos und ribeirinhos oder der seringueiros, spielten dabei keine Rolle. Sie wurden - gesellschaftlich und räumlich - im Interesse des vermeintlichen Fortschritts im wahrsten Sinne des Wortes an den Rand gedrängt. Angesichts dieser Fortschrittseuphorie fragte auch niemand, ob es eigentlich nicht genau diese Gruppen sind, die aufgrund ihrer über Generationen gesammelten Erfahrungen am ehesten wissen, wie man mit dem fragilen Naturraum Amazonien umgehen muss. Denn nach kürzester Zeit zeigten sich bereits die Kosten des neuen Entwicklungsbooms: Waldzerstörung, Bodendegradierung, Biodiversitätsverlust. Im Vergleich zu einer über Jahrhunderte verlaufenden Co-Evolution zwischen Natur und Mensch, die sich den besonderen Verhältnissen anzupassen versuchte, beherrschen nun Unterwerfung der Natur und ihre rücksichtslose Ausbeutung das Geschehen.

In jüngerer Zeit beginnt man auch in Amazonien wieder verstärkt nach Alternativen zum kompromisslosen Modernisierungskurs zu suchen und dabei auch die Erfahrungen der Völker des Waldes ernst zu nehmen. Dies ist Teil der Suche nach Pfaden einer nachhaltigen Entwicklung, die spätestens seit der Weltumweltkonferenz von Rio de Janeiro 1992 zunehmend den Diskurs um Regionalentwicklung im größten Regenwaldgebiet der Erde mitbestimmt. Amazonien hat nachgerade eine emblematische Bedeutung bei der globalen Suche nach nachhaltigen Entwicklungsalternativen erhalten.

All dies sind Facetten einer spannenden und spannungsgeladenen Geschichte, die Amazonien im Kontext des Globalen Wandels zu erzählen hat. Dabei ist seit jeher das global-lokale Wechselspiel höchst ambivalent 
je nachdem, von welcher Seite aus man es betrachtet. Aus der Sicht Brasiliens und Amazoniens ist seit Generationen das Thema der 'Internationalisierung' Amazoniens virulent. Lange Zeit von der zentralstaatlichen Obhut aufgrund der enormen Entfernungen 'abgekoppelt', schien Vielen der internationale Einfluss in Amazonien zu groß und zu unkontrollierbar. Die jüngere Geschichte liefert hierzu vermeintlich zahlreiche Belege: der Schmuggel von Setzlingen des Kautschukbaums aus Amazonien heraus, Henry Fords amazonisches Abenteuer mit der Anlage von Kautschukplantagen am Rio Tapajós, die Pläne des US-amerikanischen Futurologen Hermann Kahn zur Umgestaltung Amazoniens in einen riesigen Energieproduzenten für die Welt, die gigantischen Projekte des Milliardärs Daniel Keith Ludwig am Rio Jari und heute die Suche nach neuen Stoffen für die Kosmetikbranche, nach wertvollen Substanzen für die Pharmaindustrie, nach gentechnologisch Verwertbarem für die Agrokonzerne, der nur allzu oft der Geruch der 'Biopiraterie' anhaftet. Auch die weltweit geführten Kampagnen und das lokale Engagement der großen globalen Umweltund Menschenrechtsorganisationen für die ökologische Erhaltung Amazoniens und den Schutz seiner traditionellen Bewohner werden von vielen Brasilianern als neue Form des Imperialismus, eines Öko-Imperialismus eben, angesehen oder zumindest so in ihren ganz häufig immer noch nationalistisch ausgerichteten Diskursen über Amazonien instrumentalisiert. Über lange Zeit reagierten der Zentralstaat und insbesondere die Militärs mit einer Überwachungs-Paranoia, die sich in Truppenstationierungen und hochmodernen satellitengestützten Überwachungssystemen niederschlugen.

Und wie ist vor diesem Hintergrund die Stellung Amazoniens in den internationalen Debatten zu anthropogenem Klimawandel, zu globalem Klimaregime, zu Klimaschutz und Klimaanpassung sowie entsprechenden Governance-Strukturen zu sehen? Von einem möglichen 'Umkippen' der Ökosysteme Amazoniens im Zusammenhang des Klimawandels ist die Rede: Amazonien als einer der global bedeutsamen 'Tipping Points'. Spätestens seit der Klimakonferenz von Bali im Jahr 2007 geht es in den Diskussionen zum globalen Klimaregime in allererster Linie um die bessere Einbeziehung der großen Schwellenländer (sic Brasilien), um die Funktion der Wälder und des Waldschutzes im Klimawandel (sic Amazonien). Kürzel wie REDD und REDD+ haben Eingang in die Alltagssprache von Unternehmern, Planern und Politikern bis hinunter auf die lokale Ebene gefunden. Wenn man allerdings genauer hinschaut, dann erscheint 
das Narrativ von Amazonien in Zeiten des globalen Klimawandels nur auf den ersten Blick neu. Auf den zweiten entdeckt man eine Vielzahl von Elementen des alten Diskurses von der 'Internationalisierung' Amazoniens.

Die Geschichte von Amazonien im Globalen Wandel ist vor allem mit den aktuellen Widersprüchen hinsichtlich der Funktionen verbunden, die die unterschiedlichen Akteure - Politiker, Planer, Rinderzüchter, Energieund Bergbauunternehmen, Kleinbauern, Indigene, Umweltaktivisten, um nur einige zu nennen - der Region zuweisen. Ist Amazonien vor allem wie eh und je die Ressourcenfrontier, die mit Rohstoffen, Energie und vor allem mit ihren unermesslichen Landreserven zur nationalen Wertschöpfung beitragen soll? Dann muss die Priorität folgerichtig auf dem weiteren Ausbau der Infrastrukturen liegen, damit die Region besser als bisher in die globalen Wertschöpfungsketten einbezogen werden kann. Dass dieser modernisierungsorientierte Diskurs noch lange nicht aus den Regionalpolitiken und den regionalen Entwicklungsstrategien für Amazonien verschwunden ist, zeigen die Prioritäten des Plans zur Wachstumsbeschleunigung (PAC): Ausbau der Straßen, neue Wasserkraftwerke, Implementierung von Wasserstraßen usw. Die Inkorporation Amazoniens steht also nach wie vor auf der Tagesordnung, und zwar sowohl in die nationalen Logiken von Wachstum und Fortschritt als auch und in zunehmendem Maße in die Logiken globalisierter Wirtschaftskreisläufe. Die Frage "Wem gehört Amazonien?" ist also schon zu stellen. Wie sieht es angesichts des anhaltenden Ressourcenhungers, mit dem Amazonien konfrontiert ist, mit den Rechten der 'Völker des Waldes' aus? Welche Realisierungschancen haben die Überlebensinteressen der Kleinbauern, der Landlosen, der Verdrängten? Wo stehen Waldschutz und die Bemühungen um eine angepasste, sozial und ökologisch verträgliche Nutzung, um nachhaltige Entwicklung also? Einfache Antworten gibt es auf diese Fragen nicht, denn sehr viel hat sich in Amazonien seit dem Ende des 20. Jahrhunderts verändert. Interessen werden zunehmend und wahrnehmbar artikuliert, zahlreiche lokale Initiativen zeigen alternative Entwicklungsperspektiven auf, Netzwerke der Kooperation ziehen sich über die Region. Amazonien ist heute mehr denn je eine Region der Widersprüche. Globalisierungsdruck und Inkorporation auf der einen, vielfältige Antworten im Sinne von lokaler Selbstbestimmung, von Anpassung und nachhaltiger Entwicklung auf der anderen Seite. Amazonien ist nach wie vor eine Region der Konflikte: der handfesten Konflikte um Verfügungsrechte über Land und andere Res- 
sourcen, aber auch der Konflikte zwischen den handlungsleitenden Wahrnehmungen hinsichtlich der regionalen Potenziale und Begrenzungen neues Eldorado oder 'Grüne Hölle', Region unbegrenzter Möglichkeiten oder zu schützendes Gebiet. Die Bandbreite der Ansprüche an Amazonien zwischen globalen Interessen und lokalen Bedürfnissen ist groß.

\section{Der Mittelwesten: Vom Hinterland zur 'globalisierten Region'}

Wenn es in den letzten Jahrzehnten in Brasilien eine Region gab, die durch Einflüsse der Globalisierung ihr Profil verändert hat, dann ist es der Mittelwesten. Bis in die 1960er Jahre hinein traf auf die meisten Teilregionen des Mittelwestens, zu dem offiziell die Bundesstaaten Goiás, Mato Grosso, Mato Grosso do Sul und der Bundesdistrikt von Brasília gezählt werden (naturräumlich und strukturell gäbe es auch gute Gründe den, zumindest in der statistischen Lesart zur Nordregion zählenden Bundesstaat Tocantins ebenfalls zum Mittelwesten zu rechnen), der Begriff des interior, des Hinterlandes, in besonderer Weise zu. In den unermesslichen Weiten der Campos cerrados, der Baumsavannen, die das charakteristische Ökosystem des zentralbrasilianischen Berglandes bilden, herrschte über Generationen eine extensive Form der großbetrieblichen Rinderweidewirtschaft vor, darin quasi eingelagert Gebiete eines subsistenzorientierten Kleinbauerntums beziehungsweise Orte, die teilweise schon seit dem 18. Jahrhundert vom manuellen kleinbetrieblichen Abbau (garimpo) von Diamanten, sonstigen Edelsteinen oder Gold lebten. In vielen Gebieten des Mittelwestens war auch die indigene Präsenz lange Zeit sehr deutlich spürbar. Viele zentralbrasilianische Regionen waren im 17. und 18. Jahrhundert von den auf der Jagd nach Sklaven und Edelmetallen aus São Paulo in das weitgehend unbekannte interior vordringenden bandeirantes erkundet und unterworfen worden. Diese Eroberungszüge der bandeirantes, kleinen Gruppen von Abenteurern und Desperados, gehören inzwischen zu den nationalen Mythen Brasiliens.

Viele Orte im Mittelwesten lebten über Generationen hinweg aufgrund der enormen Entfernungen und aufgrund der fehlenden Verkehrsanbindungen in fast vollständiger Isolation und entwickelten ihren eigenen provinziellen Lebensrhythmus. So wird aus Cuiabá, der 1721 gegründeten Hauptstadt des heutigen Bundesstaates Mato Grosso, einem ehemaligen Goldgräbernest, das zum peripheren Verwaltungssitz mit seinen im be- 
schaulichen Provinzleben aufgehenden Eliten herangewachsen war, berichtet, dass man 1889 noch Monate nach der Proklamation der Republik den Geburtstag des brasilianischen Kaisers mit dem gebührenden Zeremoniell beging, ganz einfach, weil die Nachricht von der Ausrufung der Republik noch nicht in das Herz Südamerikas vorgedrungen war. Zu weit und aufwendig war der Monate andauernde Weg von Rio de Janeiro über Buenos Aires, den Rio de la Plata und den Rio Paraguai flussaufwärts. Diese Anekdote lässt erahnen, dass man sich das frühe Brasilien in seinen enormen Ausdehnungen über lange Zeit eher als eine Art Archipel nur mehr oder weniger zusammenhängender Räume vorstellen muss. Von einem tatsächlichen Nationalstaat konnte keine Rede sein.

Im 20. Jahrhundert änderte sich dies. Vor allem änderte sich die Wahrnehmung des Mittelwestens. Auch wenn politisch, wirtschaftlich und kulturell die Musik nach wie vor in den küstennahen Zonen des Südostens oder Nordostens spielte, so galt der Mittelwesten zunehmend als Region des eigentlichen, des genuinen Brasilien. Brasilidade, wenn man so will brasilianische Identität, wurde zunehmend im rustikalen, ländlichen, puristischen Mittelwesten gesehen. Dem interior wurde mehr Aufmerksamkeit zuteil. Und dies hatte handfeste politische Konsequenzen und Auswirkungen für die Regionalentwicklung. Vor allem unter Getúlio Vargas, in den 1930er und 1940er Jahren, gewann das Hinterland in politischen Diskursen und planerischen Maßnahmen an Bedeutung. In der Entwicklung und Integration des Hinterlandes sah der Diktator einen wesentlichen Bestandteil seiner politischen Mission der Erneuerung, des Estado Novo. Es wurde eigens eine staatliche Entwicklungsagentur gegründet, die Fundação Brasil Central, deren Aufgabe es war, Infrastrukturen auszubauen, Entwicklungsimpulse zu setzen. Noch heute lässt sich dies in vielen Städten des Mittelwestens nachvollziehen, in den damals entstandenen Institutionen, in der zeitbedingten Architektur und in vielen anderen Bereichen.

Der Mittelwesten ist eine Region der Städtegründungen im Geist der Moderne. Belo Horizonte, die Hauptstadt des Bundesstaates Minas Gerais, machte zu Beginn des 20. Jahrhunderts den Anfang. Goiânia, die heutige Hauptstadt des Bundesstaates Goiás, folgte als Neugründung in den 1940er Jahren. Emblematische Bedeutung gewann Brasilia, das unter der Präsidentschaft von Juscelino Kubitschek gegründet und gebaut wurde. Etwas mehr als 50 Jahre ist es her, dass auf dem Planalto Central, einem alten Verfassungsauftrag folgend, die neue Hauptstadt Brasília entstand. Das Gemeinschaftswerk des Stadtplaners Lúcio Costa und des Architek- 
ten Oscar Niemeyer wurde nicht nur zum weltweit bedeutsamen Symbol des Städtebaus der Moderne, seine Realisierung zeugt auch von der logistischen Fähigkeit des Landes, Großprojekte von epochalen AusmaBen zu realisieren. Von Anfang an war das Megaprojekt umstritten. Viel Widerstand regte sich in den Zentren der Macht, denn wer wollte schon das angenehme Leben in der cidade maravilhosa, in Rio de Janeiro, mit dem rauen, menschenleeren und eintönigen Planalto Central tauschen? Auch wurde lange Zeit angezweifelt, ob von der neuen Hauptstadt die erhofften regionalen Entwicklungsimpulse ausgehen würden. Doch trotz aller berechtigten Kritik an der sozialräumlichen Segregation, die den Bundesdistrikt von Brasília heute kennzeichnet, ist inzwischen unbestritten, dass die Realisierung von Brasília den Auftakt für einen tief greifenden und dynamischen Regionalentwicklungsprozess darstellte.

Am Beginn stand in den 1960er Jahren der Start zu einem epochalen Straßenbauprogramm. Brasília diente als Ausgangspunkt zur Erschließung der Peripherien. Fernstraßen - zumeist von den Militärs gebaut - fraßen sich durch die Wälder und Savannen und beendeten für die meisten Teilregionen die lange Zeit der Isolation und Stagnation. Mit den Straßen kamen auch die Menschen. Landsuchende, Kolonisten, Großgrundbesitzer, Investoren, aber auch viele Glücksritter und Abenteurer. Die wichtigsten Straßenprojekte, die den Mittelwesten durchmaßen, waren die Bundesstraßen Brasilia-Belém, Brasilia-Cuiabá-Porto Velho, Cuiabá-Santarém und die Straße Barra do Garças-Marabá - alles Süd-Nord-gerichtete Achsen, die die Inkorporation der peripheren Regionen in den Zentralraum Brasiliens gewährleisteten. Auch dies kann als geostrategisches Projekt der brasilianischen Militärs angesehen werden.

Die infrastrukturelle Erschließung war Voraussetzung für die 'Inwertsetzung' der weitläufigen Hochflächen des zentralbrasilianischen Massivs. Ihr Potenzial bestand vor allem in Landreserven für eine großbetriebliche Landwirtschaft, sei es die extensive Rinderhaltung, die sich vor allem in den Übergangsbereichen zu den nördlich anschließenden tropischen Regenwäldern ausbreitete, oder sei es der modernisierte, sprich der mechanisierte, kapitalintensive und nur wenig Arbeitskraft absorbierende Ackerbau. Dieser ist heute die Grundlage der wirtschaftlichen 'Erfolgsgeschichte' des Mittelwestens. Die 'konservative Modernisierung' des Agrarsektors, die wir als die brasilianische Version der Grünen Revolution bezeichnen können, hat im Mittelwesten ihr Idealgebiet gefunden. Und mit der Sojabohne stand - nach entsprechenden Züchtungs- und Adap- 
tionserfolgen - ab den 1980er Jahren für weite Bereiche des Mittelwestens, insbesondere für den flächengrößten Bundesstaat Mato Grosso, das Produkt zur Verfügung, das den über Jahrhunderte hinweg isolierten interior auf einen Schlag in den Prototyp eines 'globalisierten Ortes' nach der Diktion von Fred Scholz umwandelte. Soja-, inzwischen auch Mais- und Baumwollfelder prägen heute in vielen Gebieten des Mittelwestens das monotone (Kultur?)Landschaftsbild soweit das Auge reicht. Respektable Städte, die vor 40 Jahren als Pioniersiedlungen in Kolonisationsprojekten überhaupt erst gegründet wurden, reihen sich perlschnurartig entlang der Fernstraßen auf. Von weitem sind sie bereits an ihren riesigen Sojalagern und Trocknungsanlagen zu erkennen, die als neue 'Landmarken' den wirtschaftlichen Boom in der Kulturlandschaft materialisieren. Einige dieser neuen Städte (Sorriso, Lucas do Rio Verde, Nova Mutum) gehören inzwischen zu den wirtschaftlich erfolgreichsten Munizipien Brasiliens.

Aber dies ist nur die eine Seite der Medaille. Auf der anderen Seite ist zu fragen, wer denn vom wirtschaftlichen Erfolg eigentlich profitiert. Das ist am ehesten wohl ein immer stärker konzentriertes Agrobusiness, inzwischen in den Händen von einigen wenigen - nationalen und vor allem multinationalen - Konzernen (zu nennen sind insbesondere die 'großen vier': ADM, Bunge, Cargill, Louis Dreyfuss), das ist eine erfolgreiche Gruppe von Farmern, die großenteils vor wenigen Jahrzehnten erst aus Südbrasilien kommend in den Mittelwesten zugewandert sind und mit ihrem wirtschaftlichen Erfolg Regionalkultur und lokale Eliten vollkommen auf den Kopf stellten. Der regionale Wirtschaftsboom war sozial immer höchst exklusiv, eine Breitenwirkung im Sinne von Beschäftigungseffekten ist vergleichsweise bescheiden. Zudem ist der regionale Wirtschaftserfolg höchst fragil. Er wird von der Preisnotierung der global vermarkteten commodities an den Börsen in Chicago und anderswo auf der Welt bestimmt, er hängt von der Preisentwicklung der Vorleistungsgüter (Landmaschinen, Saatgut, Düngemittel, Pestizide) ebenso ab wie von der Entwicklung der Transportkosten. Denn trotz aller Beschleunigung ist der Distanzfaktor nach wie vor der entscheidende Standortnachteil des Mittelwestens. Auch die ökologischen Kosten sind enorm. Die Baumsavanne der Campos cerrados - nach den amazonischen Regenwäldern das flächenmäßig zweitgrößte Biom Brasiliens - gehört wahrscheinlich zu den am stärksten unterschätzten Ökosystemen des Landes. Sowohl national als auch international stand die schleichende, aber vielerorts viel radikalere Vernichtung des Cerrado sozusagen 'im Schatten’ der Zerstörung 
der amazonischen Regenwälder. Und dies, obwohl die Biodiversität des Cerrado eine enorme Vielfalt aufweist und seine Ökosystemdienstleistungen von großer Bedeutung sind. Von den meisten Umweltaktivisten viel zu wenig beachtet, ist in den letzten drei Jahrzehnten im Interesse der Zurichtung einer ganzen Region auf die globalisierten Märkte der Cerrado gründlich beseitigt und durch etwas ersetzt worden, dass sehr viel mehr einer vom Menschen gemachten 'Produktionsmaschine' als einer Kulturlandschaft entspricht.

\section{Der Süden: Das 'andere' Brasilien}

Flächenmäßig die kleinste Region, aber doch fast doppelt so groß wie Deutschland, beherbergt der Süden mit ca. 28 Millionen Menschen gut $14 \%$ der brasilianischen Bevölkerung. Die wirtschaftlichen und sozialen Indikatoren weisen dem Süden seinen Platz unter den 'Erfolgreichen' Brasiliens zu: Mit knapp $17 \%$ der zweitgrößte Beitrag zum Bruttoinlandsprodukt, nach dem Südosten und dem Mittelwesten das dritthöchste Pro-Kopf-Einkommen, noch vor dem Südosten die beste Platzierung hinsichtlich des Indexes der menschlichen Entwicklung (HDI), geringste Säuglingssterblichkeit, mit dem Südosten zusammen geringste Analphabetenquote und geringster Anteil der Armutsbevölkerung (Einkommen unter einem salário mínimo) - all dies gibt dem Süden eine Sonderstellung. Ein 'anderes' Brasilien also. Was aber sind die Gemeinsamkeiten der drei Bundesstaaten Paraná, Santa Catarina und Rio Grande do Sul? Oder ist es ihre Vielfalt, die als vorrangiges Charakteristikum gelten kann?

Naturräumlich liegen mit Ausnahme des noch zu den Randtropen zählenden Nordens des Bundesstaates Paraná alle Teilregionen des Südens im subtropisch-gemäßigten Klimabereich. Dies führt zu stärkeren jahreszeitlichen Temperaturunterschieden, die sich auf das Nutzungspotenzial der Region auswirken. So erreicht beispielsweise der Kaffeeanbau in Paraná seine klimatisch bedingten Anbaugrenzen. Von den südlichen Ausläufern der Serra do Mar und der Mata Atlântica werden weite Bereiche des küstennahen Südens geprägt. Die Wälder dominierte einstmals die Araukarie als Charakterbaum, heute vielerorts stark zurückgedrängt. In den nördlichen Bereichen wird das Hinterland der Südregion geologisch durch weit ausgedehnte basaltische Intrusionen geprägt, was deshalb von Bedeutung ist, weil sich auf ihnen fruchtbare Böden ausbilden konnten. 
Jenseits der Gebirgszüge der Serra Gaúcha geht gen Süden die Campanha Gaúcha in die Grasländer der Pampa Uruguays und Argentiniens über.

Kulturlandschaftlich wird der Süden, der einstmals eine starke Präsenz indigener Bevölkerung hatte, vom überragenden Einfluss der europäischen Einwanderung vor allem des 19. Jahrhunderts geprägt. Durchaus im Sinne einer staatlich geförderten 'Peuplierungspolitik' zu verstehen, kamen vor allem in der zweiten Hälfte des 19. Jahrhunderts zahlreiche Migranten aus Italien, aus dem heutigen Deutschland (in hohem Maße aus den damaligen ländlichen Armutsgebieten, zum Beispiel aus dem Hunsrück oder aus Pommern) oder auch aus den Gebieten der ehemaligen Donaumonarchie (zum Beispiel aus Teilen der Ukraine und Polens, zum Teil auch aus dem österreichischen Kerngebiet) in die kaum erschlossenen ländlichen Regionen des Gebirges und der Gebirgsränder. Hier bildeten sich Pionierfronten heraus, Land wurde gerodet, es entstand eine klein- und mittelbäuerliche Landwirtschaft, die der Subsistenzsicherung, aber auch der Nahrungsmittelversorgung diente. Die jeweiligen Einwanderergruppen brachten aus ihren Herkunftsgebieten Techniken und Präferenzen mit, die sich zum Teil bis heute nachvollziehen lassen. Allerdings forderte das Leben in der 'Neuen Welt' von allen Migranten auch erhebliche Anpassungsleistungen. Neben den ländlichen Zielgebieten und der Landwirtschaft prägte die europäische Zuwanderung auch die Struktur vieler (Klein- und Mittel)Städte und dort vor allem die Entwicklung von Industrie und Gewerbe. Damit sind auch schon die - oftmals klischeehaften - Vorstellungen des Südens als dem europäischsten Teil Brasiliens - von vielen auch als Grund für den regionalen 'Erfolg' ins Feld geführt - erfüllt. Was sind nun vor diesem Hintergrund der 'Andersartigkeit' die Entwicklungspfade des Südens im 20. und 21. Jahrhundert?

Die ländlichen Räume Südbrasiliens waren in den 1960er und 1970er Jahren Ausgangspunkte des agrarstrukturellen Wandels im Zeichen der Modernisierung. Die 'Grüne Revolution' auf brasilianische Art, die sich auf eine Kombination von Agrarforschung, Diffusion von Innovationen im Landbau (neue Anbaukulturen, neue Bewirtschaftungstechniken, verstärkte Marktorientierung), Agrarberatung und Agrarkreditwesen, allerdings nicht auf eine Veränderung der agrarsozialen Verhältnisse gründete, nahm hier ihren Ausgang. Die zahlreichen Kooperativen spielten dabei eine wichtige Rolle. Insbesondere die rasche Expansion des Sojaanbaus, hochmechanisiert und auf den Export orientiert, brachte es mit sich, dass immer mehr Kleinbauern aufgaben, ihr Land verkauften und in die Städte 
beziehungsweise in die Neusiedelgebiete des Mittelwestens oder Amazoniens abwanderten. Der ländliche Raum erlebte einen raschen und tief greifenden Strukturwandel, der in erheblichem Ausmaß durch Besitzkonzentration und Bevölkerungsverlust gekennzeichnet war. Demgegenüber gibt es heute in den ländlichen Räumen Südbrasiliens immer mehr Anzeichen für neue Produktionsformen (z. B. Ansätze von ökologischer Landwirtschaft, Versuche der Förderung von Regionalprodukten etc.), für eine breiter werdende Palette von Erwerbsalternativen (z. B. ländlicher Tourismus) beziehungsweise für veränderte Lebensstile. Der Süden ist unter den Großregionen Brasiliens wohl am ehesten der Raum, in dem sich neue Formen des 'Ländlichen' beobachten lassen.

Besondere Impulse gingen in den letzten Jahrzehnten von den großen städtischen Agglomerationen Südbrasiliens aus. Hier sind insbesondere Curitiba, die Hauptstadt des Bundesstaates Paraná, und Porto Alegre, die Hauptstadt des Bundesstaates Rio Grande do Sul, zu nennen. Beide Städte sind durch ihre innovative Stadtpolitik bekannt und von zahlreichen anderen Städten im In- und Ausland zum Vorbild genommen geworden. Curitiba hat sich durch eine zukunftsorientierte Stadtentwicklungsplanung, durch innovative Politiken im Bereich des Öffentlichen Personennahverkehrs, durch neue Organisationsformen der Abfallentsorgung und -verwertung sowie durch viel beachtete Projekte der Stadterneuerung den Ruf erworben, Stadthygiene sowie städtische Umwelt- und Lebensqualität im Interesse der Bürger in Einklang zu bringen. Es gilt auf Basis einer breiten Zustimmung der lokalen Bevölkerung gemeinhin als Stadt mit der höchsten Lebensqualität in Brasilien, auch wenn sich bei genauerer Betrachtung inzwischen so etwas wie ein 'Curitiba-Mythos' beobachten lässt. Hintergrund dieser 'Erfolgsgeschichte' ist eine über Jahre hinweg effiziente Stadtpolitik und -verwaltung, geführt von Persönlichkeiten mit Weitblick sowie planerischer und administrativer Kompetenz. Ein anderes, aber nicht minder innovatives Stadtpolitikmodell nahm von Porto Alegre aus seinen Weg - inzwischen weit über Brasilien hinausgehend, sogar im Sinne eines kommunalpolitischen 'Süd-Nord-Transfers'. Es geht um das so genannte orçamento participativo, das Modell eines Bürgerbeteiligungshaushalts, bei dem alljährlich auf dem Prinzip direkter Demokratie basierend über den Investitionshaushalt der Kommune in räumlich dezentralisierter und thematisch fokussierter Form entschieden wird: Porto Alegre also als 'Hauptstadt der Partizipation' in Brasilien. Entscheidend hierfür war die langjährige kommunalpolitische Kontinuität von lokalen 
PT-Regierungen, die, allen Widerständen zum Trotz, die Umsetzung von partizipativen Politikmodellen zur Priorität erklärten. Nicht zuletzt ist Porto Alegre durch seine einschlägigen Partizipationserfahrungen zum Gründungsort der Weltsozialforen, einer der wichtigsten globalisierungskritischen Bewegungen der letzten Jahre, geworden. Auch darin manifestiert sich ein 'anderes' Brasilien, ein Brasilien des bürgerschaftlichen Aufbruchs, der sozialen und politischen Vielfalt, in dem ein Mehr an cidadania zum Symbol für steigendes Selbstbewusstsein und neue soziale Verantwortung zu werden scheint.

\section{Ein oder viele Brasilien? Ein kurzes Fazit}

Zusammenfassend basiert die Wirtschafts- und Raumentwicklung Brasiliens auf verschiedenen dominanten 'Antriebskräften'. Die entscheidenden wirtschaftlichen 'Motoren' sind ohne Zweifel Industrie und moderner Dienstleistungssektor, räumlich konzentriert vor allem auf die Achse São Paulo - Rio de Janeiro, mit Ausdehnungen nach Belo Horizonte und in das Triângulo Mineiro, beziehungsweise mit kleineren Standortkonzentrationen im Distrito Federal, in Paraná und in Santa Catarina. Modernisierte Landwirtschaft und Agroindustrie spielen eine ähnlich dominante Rolle in zwei Großregionen, die durch den Westen des Bundesstaates São Paulo und den Südwesten von Mato Grosso do Sul einerseits und durch das Expansionsgebiet des Sojaanbaus in Mato Grosso sowie in geringerem Maße im Süden von Maranhão und im Westen von Bahia andererseits gebildet werden. Neben diesen dominanten Antriebskräften sind unter anderem die folgenden Faktoren für zwar oftmals begrenztere, aber trotzdem deutlich sichtbare regionale Dynamiken verantwortlich: der Bergbau, der (weltmarktorientierte) Obstanbau in Bewässerungsoasen, der Tourismus, sei es an den Küsten oder auch im Hinterland.

Stellt sich abschließend die Frage, ob sich in den letzten 10 Jahren der Regierungen Lula und Rousseff Grundsätzliches an den sozioökonomischen und räumlichen Strukturmustern Brasiliens verändert hat. Erfolge bei der Armutsbekämpfung, im Sozialbereich, beim wirtschaftlichen Wachstum und in vielen anderen gesellschaftlichen Bereichen sind nicht zu übersehen. Die Bilanz, die die Brasilianerinnen und Brasilianer ziehen, ist überwiegend positiv. Allerdings ist nicht zu übersehen, dass die generellen entwicklungsstrategischen Orientierungen auch im Kreuzfeuer der 
Kritik stehen. Zwar ist 'diskursiv' ein Bekenntnis zu den sozialen und ökologischen Zielen der Nachhaltigkeit allenthalben zu konstatieren, bei genauerer Betrachtung der entscheidenden Verlautbarungen und vor allem der konkreten Politik wird jedoch die Persistenz einer modernisierungstheoretisch begründeten Gleichsetzung von (Regional)Entwicklung mit ökonomischem Wachstum offensichtlich. So werden die im Programm zur Wachstumsbeschleunigung PAC festgeschriebenen Maßnahmen des Infrastrukturausbaus (Fernstraßen, Energie-Großprojekte etc.) über kurz oder lang zu einer deutlichen Verschärfung der ohnehin schon bestehenden Konfliktlagen, vor allem in sensiblen Gebieten wie Amazonien, beitragen. Auch wenn Brasiliens Entwicklung der letzten Jahre durchaus als Erfolgsstory gelesen werden kann, so bleiben doch zahlreiche Probleme auch angesichts des Globalen Wandels nach wie vor ungelöst. Sie ergeben sich nicht zuletzt aus den strukturellen Disparitäten des Landes, rufen unterschiedliche regionale Dynamiken hervor und perpetuieren diese gleichzeitig. Damit steckt in der regionalen Vielfalt Brasiliens Zukunftspotenzial und Zukunftshypothek gleichermaßen.

\section{Literaturverzeichnis}

Almanaque Abril 2013. São Paulo.

AnHuf, Dieter (2010): “Kein Waldschutz ohne Klimaschutz. Die Rolle der Regenwälder Amazoniens im Kampf gegen den Klimawandel”. Geographische Rundschau 62, 9, 28-33.

CoY, Martin (2010): "Stadtentwicklung und Stadtpolitik. Sozioökonomische Fragmentierung und Beispiele zukunftsorientierter Planung”. In: Costa, Sérgio/Kohlhepp, Gerd/Nitschack, Horst/Sangmeister, Hartmut (Hg.): Brasilien heute. Geographischer Raum, Politik, Wirtschaft, Kultur. 2. vollständig neu bearbeite Auflage. Frankfurt am Main: Vervuert, 51-73.

— (2013): "Environmental Justice? Sozialökologische Konfliktkonstellationen in Amazonien”. In: Burchardt, Hans-Jürgen/Dietz, Kristina/Öhlschläger, Rainer (Hg.): Umwelt und Entwicklung im 21. Jahrbundert. Impulse und Analysen aus Lateinamerika. Baden-Baden: Nomos, 121-133.

- (2013b): "Umweltprobleme und Umweltpolitik in Brasilien". Der Bürger im Staat 1-2/2013: 48-56.

Coy, Martin/KLIngler, Michael (2011): "Pionierfronten im brasilianischen Amazonien zwischen alten Problemen und neuen Dynamiken. Das Beispiel des 'Entwicklungskorridors' Cuiabá (Mato Grosso) - Santarém (Pará)". In: Innsbrucker Jahresbericht 2008 2010, 109-129. 
Coy, Martin/Schmiтt, Tobias (2007): "Brasilien - Schwellenland der Gegensätze". Geographische Rundschau 59, 9, 30-39.

Coy, Martin/Théry, Hervé (2010): "Brasilien. Sozial- und wirtschaftsräumliche Disparitäten - regionale Dynamiken”. Geographische Rundschau 62, 9, 4-11.

ISA [Instituto Socioambiental] (2007): Almanaque Brasil Socioambiental. São Paulo: ISA.

Kohlhepp, Gerd (2010a): "Regionale Disparitäten und Regionalplanung". In: Costa, Sérgio/Kohlhepp, Gerd/Nitschack, Horst/Sangmeister, Hartmut (Hg.): Brasilien heute. Geographischer Raum, Politik, Wirtschaft, Kultur. 2. vollständig neu bearbeite Auflage. Frankfurt am Main: Vervuert, 91-109.

- (2010b): "Bevölkerungsentwicklung und -struktur". In: Costa, Sérgio/Kohlhepp, Gerd/Nitschack, Horst/Sangmeister, Hartmut (Hg.): Brasilien heute. Geographischer Raum, Politik, Wirtschaft, Kultur. 2. vollständig neu bearbeite Auflage. Frankfurt am Main: Vervuert, 33-50.

KohlHepp, Gerd/Coy, Martin (2010): "Amazonien. Vernichtung durch Regionalentwicklung oder Schutz zur nachhaltigen Nutzung?”. In: Costa, Sérgio/Kohlhepp, Gerd/ Nitschack, Horst/Sangmeister, Hartmut (Hg.): Brasilien heute. Geographischer Raum, Politik, Wirtschaft, Kultur. 2. vollständig neu bearbeite Auflage. Frankfurt am Main: Vervuert, 111-134.

Neuburger, Martina (2010): "Entwicklungsprobleme des ländlichen Raumes". In: Costa, Sérgio/Kohlhepp, Gerd/Nitschack, Horst/Sangmeister, Hartmut (Hg.): Brasilien beute. Geographischer Raum, Politik, Wirtschaft, Kultur. 2. vollständig neu bearbeite Auflage. Frankfurt am Main: Vervuert, 75-89.

Sснмітт, Tobias (2010): “'O Sertão vai virar mar’. Wasser als Schlüssel der Inwertsetzungsstrategien im Nordosten Brasiliens”. Geographische Rundschau 62, 9, 12-19.

Théry, Hervé/de Mello, Neli Aparecida (2005): Atlas do Brasil. Disparidades e Dinâmicas do Território. São Paulo: Edusp. 